\title{
Modified scattering for the nonlinear nonlocal Schrödinger equation in one-dimensional case
}

Nakao Hayashi(i) and Pavel I. Naumkin

\begin{abstract}
We study the large time asymptotics of solutions to the Cauchy problem for the nonlinear nonlocal Schrödinger equation with critical nonlinearity

$$
\left\{\begin{array}{l}
i \partial_{t}\left(u-\partial_{x}^{2} u\right)+\partial_{x}^{2} u-a \partial_{x}^{4} u=\lambda|u|^{2} u, t>0, x \in \mathbb{R}, \\
u(0, x)=u_{0}(x), x \in \mathbb{R}
\end{array}\right.
$$

where $a>\frac{1}{5}, \lambda \in \mathbb{R}$. We continue to develop the factorization techniques which was started in papers Hayashi and Naumkin (Z Angew Math Phys 59(6):1002-1028, 2008) for Klein-Gordon, Hayashi and Naumkin (J Math Phys 56(9):093502, 2015) for a fourth-order Schrödinger, Hayashi and Kaikina (Math Methods Appl Sci 40(5):1573-1597, 2017) for a third-order Schrödinger to show the modified scattering of solutions to the equation. The crucial points of our approach presented here are based on the $\mathbf{L}^{2}$-boundedness of the pseudodifferential operators.
\end{abstract}

Mathematics Subject Classification. 35B40, 35Q92.

Keywords. Cubic nonlinear Schrödinger equation, Modified scattering, Decay estimates, Nonlocal Schrödinger equation.

\section{Introduction}

We study the large time asymptotics of solutions to the Cauchy problem for the nonlinear nonlocal Schrödinger equation with a critical nonlinearity in one-dimensional case

$$
\left\{\begin{array}{l}
i \partial_{t}\left(u-\partial_{x}^{2} u\right)+\partial_{x}^{2} u-a \partial_{x}^{4} u=\lambda|u|^{2} u, t>0, x \in \mathbb{R}, \\
u(0, x)=u_{0}(x), x \in \mathbb{R}
\end{array}\right.
$$

where $a>\frac{1}{5}, \lambda \in \mathbb{R}$. Equation (1.1) can be considered as a particular form of the higher-order nonlinear Schrödinger equation introduced by [30] to describe the nonlinear propagation of pulses through optical fibers. Also it arises in the context of high-speed soliton transmission in long-haul optical communication system [12]. Equation (1.1) represents the propagation of pulses by taking higher dispersion effects into account than those given by the Schrödinger equation (see $[14,22,27,31,34]$ ).

Multiplying Eq. (1.1) by the operator $\left(1-\partial_{x}^{2}\right)^{-1}$, we rewrite it in the pseudodifferential form

$$
\left\{\begin{array}{l}
i \partial_{t} u-\boldsymbol{\Lambda} u=\lambda\left(1-\partial_{x}^{2}\right)^{-1}\left(|u|^{2} u\right), t>0, x \in \mathbb{R}, \\
u(0, x)=u_{0}(x), x \in \mathbb{R},
\end{array}\right.
$$

where the linear pseudodifferential operator $\boldsymbol{\Lambda}=\left(1-\partial_{x}^{2}\right)^{-1}\left(-\partial_{x}^{2}+a \partial_{x}^{4}\right)$ is characterized by its symbol $\Lambda(\xi)=\frac{\xi^{2}+a \xi^{4}}{1+\xi^{2}}$.

As far as we know, there are no results on the large time asymptotics of solutions of the Cauchy problem (1.1). The difficulty of the small data scattering problem lies in the slow time decay rate of the $\mathbf{L}^{\infty}$-norm of solutions to the linear problem. So the problem on the large time asymptotic behavior becomes more 
difficult for low space dimensions and low order of the nonlinearity. Comparing the time decay rates of the main term and the remainder terms of the nonlinearity in (1.1), we find that this equation represents critical behavior for large time. Indeed, below we will prove the modified scattering of solutions to (1.1 ). On the other hand, the higher-order nonlinear local or homogeneous Schrödinger equations have been widely studied recently. For the local and global well-posedness of the Cauchy problem, we refer to $[4,5,29]$ and references cited therein. The dispersive blow-up was obtained in [1], [2]. The Cauchy problem for the higher-order nonlinear Schrödinger equations was intensively studied by many authors. The existence and uniqueness of solutions to (1.1) were proved in $[15,23-26,28,33]$, and the smoothing properties of solutions were studied in $[8,10,11,23-26]$.

In the case of $a=1$, we find that $\boldsymbol{\Lambda}=-\partial_{x}^{2}$, and (1.2) is the well-known cubic nonlinear Schrödinger equation studied by many authors, see $[6,17]$ and references cited therein. So we exclude the case $a=1$ from our consideration here. Final value problem for (1.2) with $a=1$ was studied by Ozawa [32], where the modified wave operator was constructed. Modified scattering of the initial value problem (1.2) with $a=1$ was obtained in [16] by using $M D \mathcal{F} M$ decomposition of the free Schrödinger evolution group $U_{1}(t)$ $(a=1)$ called as the factorization techniques. More precisely, we have the identity

$$
\begin{aligned}
U_{1}(t) \phi & =\frac{1}{\sqrt{4 \pi i t}} \int_{\mathbb{R}} e^{-\frac{(x-y)^{2}}{4 i t}} \phi(y) \mathrm{d} y=\frac{1}{\sqrt{4 \pi i t}} e^{-\frac{x^{2}}{4 i t}} \int_{\mathbb{R}} e^{-i \frac{x y}{t}} e^{-\frac{y^{2}}{4 i t}} \phi(y) \mathrm{d} y \\
& =M D \mathcal{F} M \phi,
\end{aligned}
$$

where $M=e^{-\frac{x^{2}}{4 i t}}$ is called the multiplication factor, $D \phi=\frac{1}{\sqrt{2 i t}} \phi\left(\frac{x}{t}\right)$ is called the dilation operator and $\mathcal{F}$ is the Fourier transformation defined by $\mathcal{F} \phi=\frac{1}{\sqrt{2 \pi}} \int_{\mathbb{R}} e^{-i x y} \phi(y) \mathrm{d} y$. The identity $U_{1}(t)=M D \mathcal{F} M$ was used in [21] to state the relation between the operators $x+2 i t \partial_{x}, U_{1}(t) x U_{1}(-t)$ and $M i t \partial_{x} \bar{M}$. In [16], we have used the identity

$$
\begin{aligned}
\mathcal{F} U_{1}(-t)|u|^{2} u & =\mathcal{F} U_{1}(-t)\left|U_{1}(t) U_{1}(-t) u\right|^{2} U_{1}(t) U_{1}(-t) u \\
& =\mathcal{F} \bar{M} \mathcal{F}^{-1} D^{-1} \bar{M}\left|M D \mathcal{F} M U_{1}(-t) u\right|^{2} M D \mathcal{F} M U_{1}(-t) u \\
& =\frac{1}{2 t} \mathcal{F} \bar{M} \mathcal{F}^{-1}\left|\mathcal{F} M U_{1}(-t) u\right|^{2} \mathcal{F} M U_{1}(-t) u
\end{aligned}
$$

to decompose the cubic nonlinearity in the form of the sum of the main and the remainder terms

$$
\frac{1}{2 t}|\varphi|^{2} \varphi+R, \text { with } \varphi=\mathcal{F} U(-t) u
$$

and it was shown in [16] that $R$ is the remainder term with respect to time decay. However, this method cannot be applied directly to other dispersive equations since we do not know an explicit representation for the free evolution group. Later we have used the decomposition of free evolution group (also called the factorization techniques) in the case of the cubic nonlinear Klein-Gordon equation [18], the inhomogeneous fourth-order Schrödinger equation [19] and the third-order Schrödinger equation [20]. In the present paper, our purpose is to develop this approach (the factorization techniques) for the case of the evolution group $U_{a}(t)$ generated by the nonlocal operator with symbol $\Lambda(\xi)=\frac{\xi^{2}+a \xi^{4}}{1+\xi^{2}}$, in order to find the large time asymptotic behavior of solutions. We will decompose the cubic nonlinear term into the main part and a remainder term

$$
\mathcal{F} U_{a}(-t)|u|^{2} u-\frac{1}{2 t}\left|\varphi_{a}\right|^{2} \varphi_{a}
$$

where $\varphi_{a}=\mathcal{F} U_{a}(-t) u$. In order to prove desired estimates, we use the $\mathbf{L}^{2}$ - boundedness of the pseudodifferential operators. This is a crucial point of our approach presented here. Our method can be applied to higher-dimensional cases which will be considered in a separate paper. 
To state our results precisely, we introduce Notation and Function Spaces. $\mathbf{L}^{p}=\left\{\phi \in \mathbf{S}^{\prime} ;\|\phi\|_{\mathbf{L}^{p}}<\infty\right\}$ is the usual Lebesgue space with norm $\|\phi\|_{\mathbf{L}^{p}}=\left(\int_{\mathbb{R}}|\phi(x)|^{p} \mathrm{~d} x\right)^{\frac{1}{p}}$ for $1 \leq p<\infty$ and $\|\phi\|_{\mathbf{L}^{\infty}}=$ $\sup _{x \in \mathbb{R}}|\phi(x)|$ for $p=\infty$. The weighted Sobolev space is

$$
\mathbf{H}_{p}^{m, s}=\left\{\varphi \in \mathbf{S}^{\prime} ;\|\phi\|_{\mathbf{H}_{p}^{m, s}}=\left\|\langle x\rangle^{s}\left\langle i \partial_{x}\right\rangle^{m} \phi\right\|_{\mathbf{L}^{p}}<\infty\right\},
$$

with $m, s \in \mathbb{R}, 1 \leq p \leq \infty,\langle x\rangle=\sqrt{1+x^{2}},\left\langle i \partial_{x}\right\rangle=\sqrt{1-\partial_{x}^{2}}$. Below $\mathcal{F}$ stands for the Fourier transform $\hat{\phi}(\xi)=\frac{1}{\sqrt{2 \pi}} \int_{\mathbb{R}} e^{-i x \xi} \phi(x) \mathrm{d} x$, and $\mathcal{F}^{-1}$ is the inverse Fourier transformation $\mathcal{F}^{-1} \phi=\frac{1}{\sqrt{2 \pi}} \int_{\mathbb{R}} e^{i x \xi} \phi(\xi) \mathrm{d} \xi$. We also use the notations $\mathbf{H}^{m, s}=\mathbf{H}_{2}^{m, s}, \mathbf{H}^{m}=\mathbf{H}^{m, 0}$. Let $\mathbf{C}(\mathbf{I} ; \mathbf{B})$ be the space of continuous functions from the time interval $\mathbf{I}$ to a Banach space B. Define the free evolution group $\mathcal{U}(t)=\mathcal{F}^{-1} e^{-i t \Lambda(\xi)} \mathcal{F}$, we redefine the dilation operator $\mathcal{D}_{t} \phi(x)=t^{-\frac{1}{2}} \phi\left(\frac{x}{t}\right)$, the scaling operator $(\mathcal{B} \phi)(x)=\phi(\mu(x))$, and the multiplication factor $M=e^{-i t\left(\Lambda(\eta)-\eta \Lambda^{\prime}(\eta)\right)}$, where $\mu(x)$ is defined as a root of equation

$$
\Lambda^{\prime}(\xi)=\frac{2 \xi\left(1+2 a \xi^{2}+a \xi^{4}\right)}{\left(1+\xi^{2}\right)^{2}}=x .
$$

We note here that $M$ is different from the one used previously in (1.3). We use the same notation for simplicity.

We are now in a position to state the main result of this paper.

Theorem 1.1. Let the initial data $u_{0} \in \mathbf{H}^{1} \cap \mathbf{H}^{0,1}$ and a $>\frac{1}{5}$. Assume that the norm $0<\left\|u_{0}\right\|_{\mathbf{H}^{1} \cap \mathbf{H}^{0,1}} \leq \varepsilon$. Then, there exists an $\varepsilon$ such that (1.1) has a unique global solution $u \in \mathbf{C}\left([0, \infty) ; \mathbf{H}^{1} \cap \mathbf{H}^{0,1}\right)$. Moreover, there exists a unique modified scattering state $W_{+} \in \mathbf{L}^{\infty}$ such that the asymptotics

$$
u(t)=\mathcal{D}_{t} \mathcal{B} M \frac{W_{+}}{\sqrt{i \Lambda^{\prime \prime}}} e^{-\frac{i \lambda}{\langle\xi\rangle^{2} \Lambda^{\prime \prime}}\left|W_{+}\right|^{2} \log t}+O\left(t^{-\frac{1}{2}-\delta}\right)
$$

is valid for $t \rightarrow \infty$ uniformly with respect to $x \in \mathbb{R}$, where $\delta>0$.

Remark 1.1. Large time asymptotics (1.4) can be written more explicitly in the following form

$$
\begin{aligned}
u(t, x)= & \frac{W_{+}\left(\mu\left(\frac{x}{t}\right)\right) e^{-i t\left(\Lambda\left(\mu\left(\frac{x}{t}\right)\right)-\mu\left(\frac{x}{t}\right) \Lambda^{\prime}\left(\mu\left(\frac{x}{t}\right)\right)\right)}}{\sqrt{i t \Lambda^{\prime \prime}\left(\mu\left(\frac{x}{t}\right)\right)}} e^{-\frac{i \lambda \log t}{\left\langle\mu\left(\frac{x}{t}\right)\right\rangle^{2} \Lambda^{\prime \prime}\left(\mu\left(\frac{x}{t}\right)\right)}\left|W_{+}\left(\mu\left(\frac{x}{t}\right)\right)\right|^{2}} \\
& +O\left(t^{-\frac{1}{2}-\delta}\right) .
\end{aligned}
$$

We note that the main term of the asymptotics differs from the corresponding linear case by the logarithmic oscillation which vanishes in the case of $\lambda=0$.

We organize the rest of our paper as follows. In Sect. 2, we formulate the factorization techniques. We prove the estimates of the defect operators in the uniform metrics and obtain the estimates for derivatives of the defect operator and its adjoint by applying the $\mathbf{L}^{2}$-boundedness results for pseudodifferential operators. We estimate the nonlinearity in Sect. 3. Section 4 is devoted to the proof of a priori estimates of solutions to the Cauchy problem (1.1) in the norm

$$
\|u\|_{\mathbf{X}_{T}}=\sup _{t \in[0, T]}\left(\|\widehat{\varphi}(t)\|_{\mathbf{L}^{\infty}}+(1+t)^{-\gamma}\|\widehat{\varphi}(t)\|_{\mathbf{H}^{1} \cap \mathbf{H}^{0,1}}\right),
$$

where $\widehat{\varphi}(t)=\mathcal{F U}(-t) u(t), \gamma>0$ is small depending on the size of the data. Finally, we prove Theorem 1.1 in Sect. 5 . 


\section{Preliminaries}

\subsection{Factorization techniques}

Denote the symbol $\Lambda(\xi)=\frac{\xi^{2}+a \xi^{4}}{1+\xi^{2}}$, then the free evolution group has the form $\mathcal{U}(t)=\mathcal{F}^{-1} e^{-i t \Lambda(\xi)} \mathcal{F}$. We have

$$
\begin{aligned}
\mathcal{U}(t) \mathcal{F}^{-1} \phi & =\mathcal{F}^{-1} e^{-i t \Lambda(\xi)} \phi(\xi)=\frac{1}{\sqrt{2 \pi}} \int_{\mathbb{R}} e^{i x \xi-i t \Lambda(\xi)} \phi(\xi) \mathrm{d} \xi \\
& =\frac{1}{\sqrt{2 \pi}} M \int_{\mathbb{R}} e^{i x \xi-i t\left(\Lambda(\xi)-\Lambda(\eta)+\eta \Lambda^{\prime}(\eta)\right)} \phi(\xi) \mathrm{d} \xi
\end{aligned}
$$

where

$$
M=e^{-i t\left(\Lambda(\eta)-\eta \Lambda^{\prime}(\eta)\right)} .
$$

By a direct calculation

$$
\Lambda^{\prime \prime}(\xi)=\frac{2\left(1+(6 a-3) \xi^{2}+3 a \xi^{4}+a \xi^{6}\right)}{\left(1+\xi^{2}\right)^{3}} .
$$

It is clear that $\Lambda^{\prime \prime}(\xi)>0$ if $a \geq \frac{1}{2}$. We consider the case $0 \leq a<\frac{1}{2}$. We put

$$
F(x)=1+(6 a-3) x+3 a x^{2}+a x^{3}, x=\xi^{2} \geq 0,
$$

then

$$
F^{\prime}(x)=(6 a-3)+6 a x+3 a x^{2}
$$

and

$$
F^{\prime}(x)<0 \text { for } 0<x<x_{a}, F^{\prime}(x)>0 \text { for } x>x_{a} \text {, and } F^{\prime}\left(x_{a}\right)=0
$$

where $x_{a}=\sqrt{\frac{1}{a}-1}-1 \geq 0$. Therefore, $F(x) \geq F\left(x_{a}\right)$ for any $x \geq 0$. By a simple computation, we have

$$
F\left(x_{a}\right)=3 a-1+\sqrt{a-a^{2}}>0
$$

if $a>\frac{1}{5}$. Therefore under the condition $a>\frac{1}{5}$, we have $\Lambda^{\prime \prime}(\xi)>0$ for all $\xi \in \mathbb{R}$. This guaranties $\Lambda^{\prime}(\xi)$ is monotone increasing function and the unique stationary point $\mu(x)$, defined as a root of equation

$$
\Lambda^{\prime}(\xi)=\frac{2 \xi\left(1+2 a \xi^{2}+a \xi^{4}\right)}{\left(1+\xi^{2}\right)^{2}}=x
$$

for all $x \in \mathbb{R}$. Hence, we have

$$
\begin{aligned}
\mathcal{U}(t) & \mathcal{F}^{-1} \phi \\
& =\frac{t^{\frac{1}{2}}}{\sqrt{2 \pi}} \mathcal{D}_{t} e^{-i t\left(\Lambda(\mu)-\mu \Lambda^{\prime}(\mu)\right)} \int_{\mathbb{R}} e^{i t x \xi-i t\left(\Lambda(\xi)-\Lambda(\mu)+\mu \Lambda^{\prime}(\mu)\right)} \phi(\xi) \mathrm{d} \xi \\
& =\frac{t^{\frac{1}{2}}}{\sqrt{2 \pi}} \mathcal{D}_{t} e^{-i t\left(\Lambda(\mu)-\mu \Lambda^{\prime}(\mu)\right)} \int_{\mathbb{R}} e^{i t \xi \Lambda^{\prime}(\mu)-i t\left(\Lambda(\xi)-\Lambda(\mu)+\mu \Lambda^{\prime}(\mu)\right)} \phi(\xi) \mathrm{d} \xi \\
& =\frac{t^{\frac{1}{2}}}{\sqrt{2 \pi}} \mathcal{D}_{t} e^{-i t\left(\Lambda(\mu)-\mu \Lambda^{\prime}(\mu)\right)} \int_{\mathbb{R}} e^{-i t\left(\Lambda(\xi)-\Lambda(\mu)-(\xi-\mu) \Lambda^{\prime}(\mu)\right)} \phi(\xi) \mathrm{d} \xi
\end{aligned}
$$




$$
=\frac{t^{\frac{1}{2}}}{\sqrt{2 \pi}} \mathcal{B} \mathcal{D}_{t} M \int_{\mathbb{R}} e^{-i t\left(\Lambda(\xi)-\Lambda(\eta)-(\xi-\eta) \Lambda^{\prime}(\eta)\right)} \phi(\xi) \mathrm{d} \xi .
$$

Now we write the factorization formula $\mathcal{U}(t) \mathcal{F}^{-1} \phi=\mathcal{D}_{t} \mathcal{B} M \mathcal{Q} \phi$, where $\mathcal{D}_{t} \phi=t^{-\frac{1}{2}} \phi\left(\frac{x}{t}\right),(\mathcal{B} \phi)(x)=\phi(\mu)$, and the defect operator

$$
\mathcal{Q}(t) \phi=\frac{t^{\frac{1}{2}}}{\sqrt{2 \pi}} \int_{\mathbb{R}} e^{-i t S(\xi, \eta)} \phi(\xi) \mathrm{d} \xi
$$

with phase function $S(\xi, \eta)=\Lambda(\xi)-\Lambda(\eta)-\Lambda^{\prime}(\eta)(\xi-\eta)$. Also we need the representation for the inverse evolution group $\mathcal{F U}(-t) \phi=\mathcal{Q}^{*} \bar{M} \mathcal{B}^{-1} \mathcal{D}_{t}^{-1}$, where $\mathcal{D}_{t}^{-1} \phi=t^{\frac{1}{2}} \phi(x t), \mathcal{B}^{-1} \phi=\phi\left(\Lambda^{\prime}(\eta)\right)$ and the adjoint defect operator

$$
\mathcal{Q}^{*}(t) \phi=\frac{t^{\frac{1}{2}}}{\sqrt{2 \pi}} \int_{\mathbb{R}} e^{i t S(\xi, \eta)} \phi(\eta) \Lambda^{\prime \prime}(\eta) \mathrm{d} \eta
$$

Indeed we have

$$
\begin{aligned}
\mathcal{Q}^{*} \mathcal{Q} \phi & =\frac{t^{\frac{1}{2}}}{\sqrt{2 \pi}} \int_{\mathbb{R}} e^{i t S(\xi, \eta)}(\mathcal{Q} \phi)(\eta) \Lambda^{\prime \prime}(\eta) \mathrm{d} \eta \\
& =\frac{t}{2 \pi} \int_{\mathbb{R}} e^{i t S(\xi, \eta)} \int_{\mathbb{R}} e^{-i t S(\widetilde{\xi}, \eta)} \phi(\widetilde{\xi}) d \widetilde{\xi} \mathrm{d} \Lambda^{\prime}(\eta) \\
& =\frac{t}{2 \pi} \int_{\mathbb{R}} \int_{\mathbb{R}} e^{i t(\Lambda(\xi)-\Lambda(\widetilde{\xi}))+i t x(\xi-\widetilde{\xi})} \phi(\widetilde{\xi}) d \widetilde{\xi} \mathrm{d} x \\
& =\frac{1}{2 \pi} e^{i t \Lambda(\xi)} \int_{\mathbb{R}} \int_{\mathbb{R}} e^{i x(\xi-\widetilde{\xi})} e^{-i t \Lambda(\widetilde{\xi})} \phi(\widetilde{\xi}) d \widetilde{\xi} \mathrm{d} x \\
& =\phi(\xi) .
\end{aligned}
$$

Define the new dependent variable $\widehat{\varphi}=\mathcal{F U}(-t) u(t)$. Since $\mathcal{F U}(-t) \mathcal{L}=i \partial_{t} \mathcal{F U}(-t)$, where $\mathcal{L}=i \partial_{t}-\boldsymbol{\Lambda}$, applying the operator $\mathcal{F U}(-t)$ to Eq. (1.2), we get

$$
i \partial_{t} \widehat{\varphi}=\lambda\langle\xi\rangle^{-2} t^{-1} \mathcal{Q}^{*}\left(|v|^{2} v\right)
$$

where $v=\mathcal{Q} \widehat{\varphi}$. This is our target equation. Our function space is based on the norm defined by (1.5).

For the convenience of the reader, we now state our strategy of the proof of the theorem briefly. By using the stationary phase method and integration by parts, we estimate $v=\mathcal{Q} \widehat{\varphi}$ as follows

$$
v(\eta)=(\mathcal{Q} \widehat{\varphi}(\xi))(\eta)=\frac{1}{\sqrt{i \Lambda^{\prime \prime}(\eta)}} \widehat{\varphi}(\eta)+C t^{-\frac{1}{4}}\left\|\partial_{\xi} \widehat{\varphi}\right\|_{\mathbf{L}^{2}}
$$

in Lemma 2.1 (estimate of the defect operator). This estimate will be justified if we could be able to obtain the estimate of $\left\|\partial_{\xi} \widehat{\varphi}\right\|_{\mathbf{L}^{2}}$, which requires us to estimate the norm $\left\|\partial_{\xi} \mathcal{Q}^{*}\left(|v|^{2} v\right)\right\|_{\mathbf{L}^{2}}$ via Eq. (2.1). In Lemma 2.6 we show that

$$
\left\|\partial_{\xi} \mathcal{Q}^{*}\left(|v|^{2} v\right)\right\|_{\mathbf{L}^{2}} \leq C\left\||v|^{2} v\right\|_{\mathbf{H}^{1}} \leq C\|v\|_{\mathbf{L}^{\infty}}^{2}\|v\|_{\mathbf{H}^{1}} \text {. }
$$

In order to get the estimate of $\|\widehat{\varphi}\|_{\mathbf{L}^{\infty}}$, in Lemma 2.2 we prove the estimate of $\left\|\mathcal{Q}^{*} \phi\right\|_{\mathbf{L}^{\infty}}$ (estimate of the adjoint defect operator) with $\phi=|v|^{2} v$, such as

$$
\begin{aligned}
\left(\mathcal{Q}^{*} \phi(\eta)\right)(\xi) & =\sqrt{i \Lambda^{\prime \prime}(\xi)} \phi(\xi)+C t^{-\frac{1}{4}}\left\|\partial_{\eta} \phi\right\|_{\mathbf{L}^{2}} \\
& =\sqrt{i \Lambda^{\prime \prime}(\xi)} \phi(\xi)+C t^{-\frac{1}{4}}\|v\|_{\mathbf{L}^{\infty}}^{2}\left\|\partial_{\eta} v\right\|_{\mathbf{L}^{2}} .
\end{aligned}
$$


This can be considered as the adjoint estimate to (2.2). Furthermore we show the estimate for $\left\|\partial_{\eta} v\right\|_{\mathbf{L}^{2}}=$ $\left\|\partial_{\eta} \mathcal{Q} \widehat{\varphi}\right\|_{\mathbf{L}^{2}}$ in Lemma 2.5, given by

$$
\left\|\partial_{\eta} \mathcal{Q} \widehat{\varphi}\right\|_{\mathbf{L}^{2}} \leq C\|\widehat{\varphi}\|_{\mathbf{H}^{1}}
$$

Therefore, the crucial estimates are the following

$$
\left\|\partial_{\xi} \mathcal{Q}^{*} \phi\right\|_{\mathbf{L}^{2}} \leq C\|\phi\|_{\mathbf{H}^{1}},\left\|\partial_{\eta} \mathcal{Q} \widehat{\varphi}\right\|_{\mathbf{L}^{2}} \leq C\|\widehat{\varphi}\|_{\mathbf{H}^{1}} .
$$

In order to get these estimates, we use the $\mathbf{L}^{2}$ - boundedness of pseudodifferential operators given by Lemma 2.3 and Lemma 2.4.

\subsection{Estimate for the defect operator in the uniform norm}

Consider the kernel

$$
A(t, \eta)=\sqrt{\frac{t}{2 \pi}} \int_{\mathbb{R}} e^{-i t S(\xi, \eta)} \mathrm{d} \xi .
$$

To compute the asymptotics of $A$ for large time, we apply the stationary phase method (see [13], p. 110) to find

$$
\int_{\mathbb{R}} e^{i t g(y)} f(y) \mathrm{d} y=e^{i t g\left(y_{0}\right)} f\left(y_{0}\right) \sqrt{\frac{2 \pi}{t\left|g^{\prime \prime}\left(y_{0}\right)\right|}} e^{i \frac{\pi}{4} \operatorname{sgn} g^{\prime \prime}\left(y_{0}\right)}+O\left(t^{-\frac{3}{2}}\right)
$$

for $t \rightarrow \infty$, where the stationary point $y_{0}$ is defined by the equation $g^{\prime}\left(y_{0}\right)=0$. By virtue of formula (2.3), we get

$$
A(t, \eta)=\frac{1}{\sqrt{i \Lambda^{\prime \prime}(\eta)}}\left(1+O\left(t^{-1}\right)\right)
$$

as $t \rightarrow \infty$.

In the next lemma, we estimate the defect operator $\mathcal{Q}$ in the uniform norm.

Lemma 2.1. The estimate $|\mathcal{Q} \phi-A \phi| \leq C t^{-\frac{1}{4}}\left\|\partial_{\xi} \phi\right\|_{\mathbf{L}^{2}}$ is valid for all $t \geq 1$.

Proof. By a simple calculation, we have

$$
\mathcal{Q} \phi-A \phi=\sqrt{\frac{t}{2 \pi}} \int_{\mathbb{R}} e^{-i t S(\xi, \eta)}(\phi(\xi)-\phi(\eta)) \mathrm{d} \xi .
$$

Integration by parts via identity

$$
e^{-i t S(\xi, \eta)}=H_{1} \partial_{\xi}\left((\xi-\eta) e^{-i t S(\xi, \eta)}\right)
$$

where

$$
H_{1}=\left(1-i t(\xi-\eta) \partial_{\xi} S(\xi, \eta)\right)^{-1}
$$

yields

$$
\begin{gathered}
\mathcal{Q} \phi-A \phi=C t^{\frac{1}{2}} \int_{\mathbb{R}} e^{-i t S(\xi, \eta)}(\phi(\xi)-\phi(\eta))(\xi-\eta) \partial_{\xi} H_{1} \mathrm{~d} \xi \\
+C t^{\frac{1}{2}} \int_{\mathbb{R}} e^{-i t S(\xi, \eta)}(\xi-\eta) H_{1} \partial_{\xi} \phi(\xi) \mathrm{d} \xi
\end{gathered}
$$


Note that $\Lambda^{\prime \prime}(\xi)=O(1)$. Also we have

$$
\partial_{\xi} S(\xi, \eta)=\Lambda^{\prime}(\xi)-\Lambda^{\prime}(\eta)=(\xi-\eta) \int_{0}^{1} \Lambda^{\prime \prime}(\eta+(\xi-\eta) z) \mathrm{d} z=O(\xi-\eta) .
$$

Hence the estimate follows

$$
\left|H_{1}\right|+\left|(\xi-\eta) \partial_{\xi} H_{1}\right| \leq \frac{C}{1+t(\xi-\eta)^{2}} .
$$

Therefore by Hardy and Cauchy-Schwarz inequalities, we obtain

$$
\begin{gathered}
|\mathcal{Q} \phi-A \phi| \leq C t^{\frac{1}{2}} \int_{\mathbb{R}}\left(\frac{|\phi(\xi)-\phi(\eta)|}{|\xi-\eta|}+\left|\partial_{\xi} \phi(\xi)\right|\right) \frac{|\xi-\eta| \mathrm{d} \xi}{1+t(\xi-\eta)^{2}} \\
\quad \leq C t^{\frac{1}{2}}\left\|\partial_{\xi} \phi\right\|_{\mathbf{L}^{2}}\left(\int_{\mathbb{R}} \frac{(\xi-\eta)^{2} d \xi}{\left(1+t(\xi-\eta)^{2}\right)^{2}}\right)^{\frac{1}{2}} \leq C t^{-\frac{1}{4}}\left\|\partial_{\xi} \phi\right\|_{\mathbf{L}^{2}} .
\end{gathered}
$$

Lemma 2.1 is proved.

\subsection{Estimate for the adjoint defect operator in the uniform norm}

We consider the kernel

$$
A^{*}(t, \xi)=\sqrt{\frac{t}{2 \pi}} \int_{\mathbb{R}} e^{i t S(\xi, \eta)} \Lambda^{\prime \prime}(\eta) \mathrm{d} \eta .
$$

By virtue of formula (2.3), we obtain the large time asymptotics

$$
A^{*}(t, \xi)=\sqrt{i \Lambda^{\prime \prime}(\xi)}\left(1+O\left(t^{-1}\right)\right)
$$

for $t \rightarrow \infty$. In the next lemma, we estimate the adjoint defect operator $\mathcal{Q}^{*}$ in the uniform norm.

Lemma 2.2. The estimate $\left|\mathcal{Q}^{*} \phi-A^{*} \phi\right| \leq C t^{-\frac{1}{4}}\left\|\partial_{\eta} \phi\right\|_{\mathbf{L}^{2}}$ is valid for all $t \geq 1$.

Proof. As above, we integrate by parts via the identity

$$
e^{i t S(\xi, \eta)}=H_{2} \partial_{\eta}\left((\eta-\xi) e^{i t S(\xi, \eta)}\right)
$$

with

$$
H_{2}=\left(1+i t(\eta-\xi) \partial_{\eta} S(\xi, \eta)\right)^{-1}
$$

and $\partial_{\eta} S(\xi, \eta)=\Lambda^{\prime \prime}(\eta)(\eta-\xi)=O(\eta-\xi)$. Then, we find

$$
\begin{aligned}
\mathcal{Q}^{*} \phi & -A^{*} \phi=\sqrt{\frac{t}{2 \pi}} \int_{\mathbb{R}} e^{i t S(\xi, \eta)}(\phi(\eta)-\phi(\xi)) \Lambda^{\prime \prime}(\eta) \mathrm{d} \eta \\
= & C t^{\frac{1}{2}} \int_{\mathbb{R}} e^{i t S(\xi, \eta)} \frac{\phi(\eta)-\phi(\xi)}{\eta-\xi}(\eta-\xi)^{2} \partial_{\eta}\left(H_{2} \Lambda^{\prime \prime}(\eta)\right) \mathrm{d} \eta \\
& +C t^{\frac{1}{2}} \int_{\mathbb{R}} e^{i t S(\xi, \eta)}(\eta-\xi) H_{2} \Lambda^{\prime \prime}(\eta) \partial_{\eta} \phi(\eta) \mathrm{d} \eta
\end{aligned}
$$


From which via the estimate

$$
\left|H_{2} \Lambda^{\prime \prime}(\eta)\right|+\left|(\eta-\xi) \partial_{\eta}\left(H_{2} \Lambda^{\prime \prime}(\eta)\right)\right| \leq \frac{C}{1+t(\eta-\xi)^{2}},
$$

and Hardy and Cauchy-Schwarz inequalities, we obtain

$$
\left|\mathcal{Q}^{*} \phi-A^{*} \phi\right| \leq C t^{\frac{1}{2}}\left\|\partial_{\eta} \phi\right\|_{\mathbf{L}^{2}}\left(\int_{\mathbb{R}} \frac{(\eta-\xi)^{2} \mathrm{~d} \eta}{\left(1+t(\eta-\xi)^{2}\right)^{2}}\right)^{\frac{1}{2}} \leq C t^{-\frac{1}{4}}\left\|\partial_{\eta} \phi\right\|_{\mathbf{L}^{2}} .
$$

Lemma 2.2 is proved.

\subsection{Boundedness of pseudodifferential operators}

There are many papers devoted to the $\mathbf{L}^{2}$ estimates of pseudodifferential operators (see, e.g. [3,7,9]). Below we will use the following result on the $\mathbf{L}^{2}$ boundedness of pseudodifferential operator

$$
\mathbf{a}(x, \mathbf{D}) \phi \equiv \int_{\mathbb{R}} e^{i x \xi} \mathbf{a}(x, \xi) \widehat{\phi}(\xi) \mathrm{d} \xi
$$

$($ see $[9])$.

Lemma 2.3. Let the symbol $\mathbf{a}(x, \xi)$ be such that

$$
\sup _{x, \xi \in \mathbb{R}}\left|\partial_{x}^{k} \partial_{\xi}^{l} \mathbf{a}(x, \xi)\right| \leq C
$$

for $k, l=0,1$. Then

$$
\|\mathbf{a}(x, \mathbf{D}) \phi\|_{\mathbf{L}_{x}^{2}} \leq C\|\phi\|_{\mathbf{L}^{2}} .
$$

Analogously for the conjugate pseudodifferential operator

$$
\mathbf{a}^{*}(\xi, \mathbf{D}) \phi \equiv \int_{\mathbb{R}} e^{-i x \xi} \mathbf{a}^{*}(x, \xi) \overline{\widehat{\phi}(x)} \mathrm{d} x
$$

we get the following result.

Lemma 2.4. Let the symbol

$$
\sup _{x, \xi \in \mathbb{R}}\left|\partial_{x}^{k} \partial_{\xi}^{l} \mathbf{a}^{*}(x, \xi)\right| \leq C
$$

for $k, l=0,1$. Then

$$
\left\|\mathbf{a}^{*}(\xi, \mathbf{D}) \phi\right\|_{\mathbf{L}_{x}^{2}} \leq C\|\phi\|_{\mathbf{L}^{2}}
$$

\subsection{Estimate for derivative of the defect operator}

In the next lemma, we estimate a derivative of the defect operator $\mathcal{Q}$. 
Lemma 2.5. The estimate $\left\|\partial_{\eta} \mathcal{Q} \phi\right\|_{\mathbf{L}^{2}} \leq C\|\phi\|_{\mathbf{H}^{1}}$ is true for all $t \geq 1$.

Proof. We integrate by parts to get

$$
\partial_{\eta} \mathcal{Q} \phi=C t^{\frac{1}{2}} \int_{\mathbb{R}} e^{-i t S(\xi, \eta)}\left(q_{1}(\xi, \eta) \partial_{\xi} \phi(\xi)+q_{2}(\xi, \eta) \phi(\xi)\right) \mathrm{d} \xi,
$$

where

$$
q_{1}(\xi, \eta)=\frac{\partial_{\eta} S(\xi, \eta)}{\partial_{\xi} S(\xi, \eta)}
$$

and

$$
q_{2}(\xi, \eta)=\partial_{\xi}\left(\frac{\partial_{\eta} S(\xi, \eta)}{\partial_{\xi} S(\xi, \eta)}\right)
$$

Then, we obtain

$$
\begin{aligned}
\partial_{\eta} \mathcal{Q} \phi & =C \bar{M} \mathcal{B}^{-1} \mathcal{D}_{t}^{-1} \int_{\mathbb{R}} e^{i x \xi} q_{1}\left(\xi, \mu\left(\frac{x}{t}\right)\right) e^{-i t \Lambda(\xi)} \partial_{\xi} \phi(\xi) \mathrm{d} \xi \\
& +C \bar{M} \mathcal{B}^{-1} \mathcal{D}_{t}^{-1} \int_{\mathbb{R}} e^{i x \xi} q_{2}\left(\xi, \mu\left(\frac{x}{t}\right)\right) e^{-i t \Lambda(\xi)} \phi(\xi) \mathrm{d} \xi \\
& =C \bar{M} \mathcal{B}^{-1} \mathcal{D}_{t}^{-1} \mathbf{a}_{1}(x, \mathbf{D}) \mathcal{F}^{-1} e^{-i t \Lambda} \partial_{\xi} \phi+C \bar{M} \mathcal{B}^{-1} \mathcal{D}_{t}^{-1} \mathbf{a}_{2}(x, \mathbf{D}) \mathcal{F}^{-1} e^{-i t \Lambda} \phi
\end{aligned}
$$

where the symbols $\mathbf{a}_{1}(x, \xi)=q_{1}\left(\xi, \mu\left(\frac{x}{t}\right)\right)$ and $\mathbf{a}_{2}(x, \xi)=q_{2}\left(\xi, \mu\left(\frac{x}{t}\right)\right)$. Since

$$
\begin{gathered}
\mu(x)=O(x), \mu^{\prime}(x)=\frac{1}{\Lambda^{\prime \prime}(\mu(x))}=O(1) \\
q_{1}(\xi, \eta)=\frac{\Lambda^{\prime \prime}(\eta)}{\int_{0}^{1} \Lambda^{\prime \prime}(\eta+(\xi-\eta) z) \mathrm{d} z}=O(1)
\end{gathered}
$$

and

$$
q_{2}(\xi, \eta)=\partial_{\xi}\left(\frac{\Lambda^{\prime \prime}(\eta)}{\int_{0}^{1} \Lambda^{\prime \prime}(\eta+(\xi-\eta) z) \mathrm{d} z}\right)=O(1)
$$

we find

$$
\sup _{x, \xi \in \mathbb{R}}\left|\partial_{x}^{k} \partial_{\xi}^{l} \mathbf{a}_{j}(x, \xi)\right| \leq C \text { for } k, l=0,1, j=1,2 .
$$

Hence by Lemma 2.3, we get

$$
\left\|\mathbf{a}_{j}(x, \mathbf{D}) \phi\right\|_{\mathbf{L}_{x}^{2}} \leq C\|\phi\|_{\mathbf{L}^{2}} .
$$

Thus in view of equalities

$$
\left\|\mathcal{B}^{-1} \phi\right\|_{\mathbf{L}^{2}}=\|\phi\|_{\mathbf{L}^{2}},\left\|\mathcal{D}_{t}^{-1} \phi\right\|_{\mathbf{L}^{2}}=\|\phi\|_{\mathbf{L}^{2}}
$$

and $\left\|\mathcal{F}^{-1} \phi\right\|_{\mathbf{L}^{2}}=\|\phi\|_{\mathbf{L}^{2}}$, we obtain 


$$
\begin{aligned}
& \left\|\partial_{\eta} \mathcal{Q} \phi\right\|_{\mathbf{L}_{x}^{2}} \leq C\left\|\mathcal{B}^{-1} \mathcal{D}_{t}^{-1} \mathbf{a}_{1}(x, \mathbf{D}) \mathcal{F}^{-1} e^{-i t \Lambda} \partial_{\xi} \phi\right\|_{\mathbf{L}^{2}} \\
& +C\left\|\mathcal{B}^{-1} \mathcal{D}_{t}^{-1} \mathbf{a}_{2}(x, \mathbf{D}) \mathcal{F}^{-1} e^{-i t \Lambda} \phi\right\|_{\mathbf{L}^{2}} \leq C\left\|\partial_{\xi} \phi\right\|_{\mathbf{L}^{2}}+C\|\phi\|_{\mathbf{L}^{2}} .
\end{aligned}
$$

Lemma 2.5 is proved.

\subsection{Estimate for derivative of the adjoint defect operator}

Next we prove the estimates for derivatives of adjoint defect operator $\mathcal{Q}^{*}$.

Lemma 2.6. The estimate $\left\|\partial_{\xi} \mathcal{Q}^{*} \phi\right\|_{\mathbf{L}^{2}} \leq C\|\phi\|_{\mathbf{H}^{1}}$ is true for all $t \geq 1$.

Proof. Integrating by parts, we get

$$
\partial_{\xi} \mathcal{Q}^{*} \phi=C t^{\frac{1}{2}} \int_{\mathbb{R}} e^{i t S(\xi, \eta)}\left(q_{3}(\xi, \eta) \partial_{\eta} \phi(\eta)+q_{4}(\xi, \eta) \phi(\eta)\right) \Lambda^{\prime \prime}(\eta) \mathrm{d} \eta,
$$

where

$$
q_{3}(\xi, \eta)=\frac{\partial_{\xi} S(\xi, \eta)}{\partial_{\eta} S(\xi, \eta)}
$$

and

$$
q_{4}(\xi, \eta)=\frac{1}{\Lambda^{\prime \prime}(\eta)} \partial_{\eta}\left(\frac{\partial_{\xi} S(\xi, \eta)}{\partial_{\eta} S(\xi, \eta)} \Lambda^{\prime \prime}(\eta)\right) .
$$

Then changing the variable of integration $\eta=\mu(x)$ and after that $x=\frac{x^{\prime}}{t}$, we find

$$
\begin{gathered}
\partial_{\xi} \mathcal{Q}^{*} \phi=C e^{i t \Lambda(\xi)} \int_{\mathbb{R}} e^{-i x \xi} q_{3}\left(\xi, \mu\left(\frac{x}{t}\right)\right)\left(\mathcal{D}_{t} \mathcal{B} M \partial_{\eta} \phi\right) d x \\
+C e^{i t \Lambda(\xi)} \int_{\mathbb{R}} e^{-i x \xi} q_{4}\left(\xi, \mu\left(\frac{x}{t}\right)\right)\left(\mathcal{D}_{t} \mathcal{B} M \phi\right) \mathrm{d} x .
\end{gathered}
$$

Denote $\mathbf{a}_{3}^{*}(\xi, x)=q_{3}\left(\xi, \mu\left(\frac{x}{t}\right)\right)$ and $\mathbf{a}_{4}^{*}(\xi, x)=q_{4}\left(\xi, \mu\left(\frac{x}{t}\right)\right)$, then

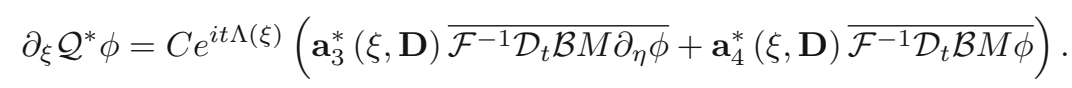

We have $\mu(x)=O(x), \mu^{\prime}(x)=\frac{1}{\Lambda^{\prime \prime}(\mu(x))}=O(1)$,

$$
q_{3}(\xi, \eta)=\frac{1}{\Lambda^{\prime \prime}(\eta)} \int_{0}^{1} \Lambda^{\prime \prime}(\eta+(\xi-\eta) z) \mathrm{d} z=O(1)
$$

and

$$
q_{4}(\xi, \eta)=\frac{1}{\Lambda^{\prime \prime}(\eta)} \partial_{\eta} \int_{0}^{1} \Lambda^{\prime \prime}(\eta+(\xi-\eta) z) \mathrm{d} z=O(1)
$$

hence

$$
\sup _{x, \xi \in \mathbb{R}}\left|\partial_{x}^{k} \partial_{\xi}^{l} \mathbf{a}_{j}^{*}(\xi, x)\right| \leq C
$$

for $k, l=0,1, j=3,4$. Application of Lemma 2.4 yields

$$
\left\|\mathbf{a}_{j}^{*}(\xi, \mathbf{D}) \phi\right\|_{\mathbf{L}_{x}^{2}} \leq C\|\phi\|_{\mathbf{L}^{2}} .
$$


Then in view of equalities $\|\mathcal{B} \phi\|_{\mathbf{L}^{2}}=\|\phi\|_{\mathbf{L}^{2}},\left\|\mathcal{D}_{t} \phi\right\|_{\mathbf{L}^{2}}=\|\phi\|_{\mathbf{L}^{2}}$ and $\left\|\mathcal{F}^{-1} \phi\right\|_{\mathbf{L}^{2}}=\|\phi\|_{\mathbf{L}^{2}}$, we find

$$
\begin{aligned}
& \left\|\partial_{\xi} \mathcal{Q}^{*} \phi\right\|_{\mathbf{L}_{x}^{2}} \leq C\left\|\mathbf{a}_{3}^{*}(\xi, \mathbf{D}) \overline{\mathcal{F}^{-1} \mathcal{D}_{t} \mathcal{B} M \partial_{\eta} \phi}\right\|_{\mathbf{L}^{2}} \\
& +C\left\|\mathbf{a}_{4}^{*}(\xi, \mathbf{D}) \overline{\mathcal{F}^{-1} \mathcal{D}_{t} \mathcal{B} M \phi}\right\|_{\mathbf{L}^{2}} \leq C\left\|\partial_{\eta} \phi\right\|_{\mathbf{L}^{2}}+C\|\phi\|_{\mathbf{L}^{2}} .
\end{aligned}
$$

Lemma 2.6 is proved.

\section{Estimates for the nonlinearity}

Define the norm

$$
\|u\|_{\mathbf{X}_{T}}=\sup _{t \in[0, T]}\left(\|\widehat{\varphi}(t)\|_{\mathbf{L}^{\infty}}+(1+t)^{-\gamma}\|\widehat{\varphi}(t)\|_{\mathbf{H}^{1} \cap \mathbf{H}^{0,1}}\right)
$$

where $\widehat{\varphi}(t)=\mathcal{F U}(-t) u(t), \gamma=C \varepsilon>0$ is small.

\subsection{Asymptotics of the nonlinearity}

In the next lemma, we calculate the asymptotic representation for the nonlinearity.

Lemma 3.1. Suppose that $\|u\|_{\mathbf{X}_{T}} \leq C \varepsilon$. Then the asymptotics

$$
\mathcal{Q}^{*}|v|^{2} v=\frac{1}{\Lambda^{\prime \prime}}|\widehat{\varphi}|^{2} \widehat{\varphi}+O\left(\varepsilon^{3} t^{\gamma-\frac{1}{4}}\right)
$$

is true for all $t \in[1, T]$, where $v=\mathcal{Q} \widehat{\varphi}, \widehat{\varphi}(t)=\mathcal{F U}(-t) u(t)$.

Proof. Applying Lemma 2.2 with $\phi=|v|^{2} v$, we find

$$
\mathcal{Q}^{*}|v|^{2} v=\sqrt{i \Lambda^{\prime \prime}}|v|^{2} v+O\left(t^{-1}\|v\|_{\mathbf{L}^{\infty}}^{3}\right)+O\left(t^{-\frac{1}{4}}\|v\|_{\mathbf{L}^{\infty}}^{2}\left\|\partial_{\eta} v\right\|_{\mathbf{L}^{2}}\right) .
$$

In view of Lemma 2.1, we have the asymptotics $v=\frac{1}{\sqrt{i \Lambda^{\prime \prime}}} \widehat{\varphi}+O\left(\varepsilon t^{\gamma-\frac{1}{4}}\right)$, and estimate $\|v\|_{\mathbf{L}^{\infty}} \leq C \varepsilon$. Then by Lemma 2.5 and condition of the lemma we find $\left\|\partial_{\eta} v\right\|_{\mathbf{L}^{2}} \leq C \varepsilon t^{\gamma}$. Hence, the result of the lemma follows. Lemma 3.1 is proved.

\subsection{Estimate for derivative of the nonlinearity in Eq. (2.1).}

Lemma 3.2. Suppose that $\|u\|_{\mathbf{X}_{T}} \leq C \varepsilon$. Then, the estimate $\left\|\partial_{\xi} \mathcal{Q}^{*}|v|^{2} v\right\|_{\mathbf{L}^{2}} \leq C \varepsilon^{3} t^{\gamma}$ is true for all $t \geq 1$, where $v=\mathcal{Q} \widehat{\varphi}, \widehat{\varphi}(t)=\mathcal{F U}(-t) u(t), \gamma=C \varepsilon$.

Proof. By virtue of Lemma 2.6 with $\phi=|v|^{2} v$, we get $\left\|\partial_{\xi} \mathcal{Q}^{*}|v|^{2} v\right\|_{\mathbf{L}^{2}} \leq C\|v\|_{\mathbf{L}^{\infty}}^{2}\|v\|_{\mathbf{H}^{1}}$. Using Lemma 2.1, we find $\|v\|_{\mathbf{L}^{\infty}} \leq C \varepsilon$. Then by Lemma 2.5, we obtain the result of the lemma. Lemma 3.2 is proved. 


\section{A priori estimate of solutions}

We state the local existence of solutions to the Cauchy problem (1.1) in the functional space $\mathbf{H}^{1} \cap \mathbf{H}^{0,1}$ which is shown by the well-known contraction mapping principle.

Theorem 4.1. Assume that the initial data $u_{0} \in \mathbf{H}^{1} \cap \mathbf{H}^{0,1}$. Then, there exists a time $T>0$, which depends on the norm $\left\|u_{0}\right\|_{\mathbf{H}^{1} \cap \mathbf{H}^{0,1}}$, such that (1.1) has a unique solution $\mathcal{U}(-t) u \in \mathbf{C}\left([0, T] ; \mathbf{H}^{1} \cap \mathbf{H}^{0,1}\right)$ such that $\|u\|_{\mathbf{x}_{T}}<C$. If the norm $\left\|u_{0}\right\|_{\mathbf{H}^{1} \cap \mathbf{H}^{0,1}}$ is small, then the existence time $T \geq 1$.

To prove the global result, we need a priori estimate of the norm $\|u\|_{\mathbf{X}_{T}}$ uniformly with respect to $T \geq 1$.

Lemma 4.1. Let the initial data $u_{0} \in \mathbf{H}^{1} \cap \mathbf{H}^{0,1}$ have a small norm $\left\|u_{0}\right\|_{\mathbf{H}^{1} \cap \mathbf{H}^{0,1}}$. Then, the estimate $\|u\|_{\mathbf{X}_{T}}<C \varepsilon$ is true for all $T \geq 1$.

Proof. Arguing by the contradiction, we can find the first moment of time $T>0$, such that $\|u\|_{\mathbf{X}_{T}}=C \varepsilon$. By Lemma 3.1 and (2.1) we get

$$
i \partial_{t} \widehat{\varphi}=\frac{\lambda}{t\langle\xi\rangle^{2} \Lambda^{\prime \prime}}|\widehat{\varphi}|^{2} \widehat{\varphi}+O\left(\langle\xi\rangle^{-2} \varepsilon^{3} t^{\gamma-\frac{5}{4}}\right) .
$$

Multiplying (4.1) by $\bar{\varphi}$ and taking the real part of the result, we get

$$
\partial_{t}|\widehat{\varphi}|^{2}=O\left(\langle\xi\rangle^{-2} \varepsilon^{3} t^{\gamma-\frac{5}{4}}\right)|\widehat{\varphi}|
$$

from which it follows that

$$
\partial_{t}|\widehat{\varphi}|=O\left(\langle\xi\rangle^{-2} \varepsilon^{3} t^{\gamma-\frac{5}{4}}\right) .
$$

Hence integration in time yields $|\widehat{\varphi}(t, \xi)| \leq|\widehat{\varphi}(0, \xi)|+C \varepsilon^{3}<C \varepsilon$ for all $t \in[1, T]$. Next we estimate the norm $\|\widehat{\varphi}\|_{\mathbf{H}^{1}}$. We have from $(2.1)$

and by Lemma 3.2

$$
\frac{\mathrm{d}}{\mathrm{d} t}\|\widehat{\varphi}(t)\|_{\mathbf{H}^{1}}^{2} \leq C t^{-1}\left\|\mathcal{Q}^{*}|v|^{2} v\right\|_{\mathbf{H}^{1}}\|\widehat{\varphi}(t)\|_{\mathbf{H}^{1}}
$$

$$
\frac{\mathrm{d}}{\mathrm{d} t}\|\widehat{\varphi}(t)\|_{\mathbf{H}^{1}} \leq C t^{-1}\left\|\mathcal{Q}^{*}|v|^{2} v\right\|_{\mathbf{H}^{1}} \leq C \varepsilon^{3} t^{\gamma-1}
$$

Then integrating in time, we get $\|\widehat{\varphi}(t)\|_{\mathbf{H}^{1}} \leq\|\widehat{\varphi}(0)\|_{\mathbf{H}^{1}}+C \varepsilon^{3} t^{\gamma}<C \varepsilon t^{\gamma}$ for all $t \in[1, T]$. Also multiplying (1.1) by $\bar{u}$ and taking the imaginary part of the result we have

$$
\frac{\mathrm{d}}{\mathrm{d} t}\left(\|u(t)\|_{\mathbf{L}^{2}}+\left\|\partial_{x} u(t)\right\|_{\mathbf{L}^{2}}\right)=0 .
$$

Therefore, integration in time gives us $\|\widehat{\varphi}(t)\|_{\mathbf{H}^{0,1}}=\|u(t)\|_{\mathbf{H}^{1}} \leq\left\|u_{0}\right\|_{\mathbf{H}^{1}}<C \varepsilon$. Thus, we get $\|u\|_{\mathbf{X}_{T}}<C \varepsilon$ for all $T>1$. We obtain the desired contradiction. Lemma 4.1 is proved.

\section{Proof of Theorem 1.1}

By Lemma 4.1, we see that a priori estimate of the norm $\|u\|_{\mathbf{X}_{T}} \leq C \varepsilon$ is true for all $T>0$. Therefore, the global existence of solutions of the Cauchy problem (1.1) satisfying estimate $\|u\|_{\mathbf{X}_{\infty}} \leq C \varepsilon$ follows by a standard continuation argument and the local existence Theorem 4.1. Now we turn to the proof of the asymptotic formula (1.4) for the solutions $u$ of the Cauchy problem (1.1). As in the proof of Lemma 4.1, we obtain Eq. (4.1)

$$
\partial_{t} \widehat{\varphi}=-\frac{i \lambda}{t\langle\xi\rangle^{2} \Lambda^{\prime \prime}(\xi)}|\widehat{\varphi}|^{2} \widehat{\varphi}+O\left(\varepsilon^{3} t^{\gamma-\frac{5}{4}}\right)
$$


Changing

$$
g(t, \xi)=\widehat{\varphi}(t, \xi) \exp \left(\frac{i \lambda}{\langle\xi\rangle^{2} \Lambda^{\prime \prime}(\xi)} \int_{1}^{t}|\widehat{\varphi}(\tau, \xi)|^{2} \frac{d \tau}{\tau}\right),
$$

we get $\partial_{t} g=O\left(\varepsilon^{3} t^{-1-\delta}\right)$, where $\delta=\frac{1}{4}-\gamma$. Integrating in time, we find

$$
\|g(t)-g(s)\|_{\mathbf{L}^{\infty}} \leq C \varepsilon \int_{s}^{t} \tau^{-1-\delta} \mathrm{d} \tau \leq C \varepsilon s^{-\delta}
$$

for all $t>s>0$. Therefore, there exists a unique final state $g_{+} \in \mathbf{L}^{\infty}$, such that

$$
\left\|g(t)-g_{+}\right\|_{\mathbf{L}^{\infty}} \leq C \varepsilon t^{-\delta}
$$

for all $t>0$. Denote

$$
\Phi(t)=\int_{1}^{t}|g(\tau)|^{2} \frac{d \tau}{\tau}-\left|g_{+}\right|^{2} \log t
$$

We have

$$
\Phi(t)-\Phi(s)=\int_{s}^{t}\left(|g(\tau)|^{2}-|g(t)|^{2}\right) \frac{d \tau}{\tau}+\left(|g(t)|^{2}-\left|g_{+}\right|^{2}\right) \log \frac{t}{s} .
$$

Hence,

$$
\|\Phi(t)-\Phi(s)\|_{\mathbf{L}^{\infty}} \leq C \varepsilon^{2} s^{-\delta}
$$

for all $t>s>0$. Thus, there exists a unique real-valued function $\Phi_{+}$, such that $\Phi_{+} \in \mathbf{L}^{\infty}$ and

$$
\left\|\Phi(t)-\Phi_{+}\right\|_{\mathbf{L}^{\infty}} \leq C \varepsilon^{2} t^{-\delta} .
$$

Therefore, we obtain

$$
\int_{1}^{t}|\widehat{\varphi}(\tau)|^{2} \frac{d \tau}{\tau}=\Phi(t)+\left|g_{+}\right|^{2} \log t=\Phi_{+}+\left|g_{+}\right|^{2} \log t+O\left(\varepsilon t^{-\delta}\right) .
$$

Then, we find the asymptotics

$$
\begin{aligned}
\widehat{\varphi}(t) & =g_{+} \exp \left(-\frac{i \lambda}{\langle\xi\rangle^{2} \Lambda^{\prime \prime}(\xi)} \int_{1}^{t}|\widehat{\varphi}(\tau)|^{2} \frac{d \tau}{\tau}\right)+O\left(t^{-\delta}\right) \\
& =g_{+} \exp \left(-\frac{i \lambda}{\langle\xi\rangle^{2} \Lambda^{\prime \prime}}\left|g_{+}\right|^{2} \log t-\frac{i \lambda}{\langle\xi\rangle^{2} \Lambda^{\prime \prime}} \Phi_{+}\right)+O\left(t^{-\delta}\right) \\
& =W_{+} \exp \left(-\frac{i \lambda}{\langle\xi\rangle^{2} \Lambda^{\prime \prime}}\left|W_{+}\right|^{2} \log t\right)+O\left(t^{-\delta}\right)
\end{aligned}
$$

where

$$
W_{+}=g_{+} \exp \left(-\frac{i \lambda}{\langle\xi\rangle^{2} \Lambda^{\prime \prime}} \Phi_{+}\right) \in \mathbf{L}^{\infty}
$$


Finally using the factorization formulas for $\mathcal{U}(t)$ and the result of Lemma 2.1, we have

$$
u(t)=\mathcal{D}_{t} \mathcal{B} M \mathcal{Q} \widehat{\varphi}=\mathcal{D}_{t} \mathcal{B} M \frac{W_{+}}{\sqrt{i \Lambda^{\prime \prime}}} \exp \left(-\frac{i \lambda}{\langle\xi\rangle^{2} \Lambda^{\prime \prime}}\left|W_{+}\right|^{2} \log t\right)+O\left(t^{-\frac{1}{2}-\delta}\right) .
$$

This completes the proof of asymptotics (1.4). Theorem 1.1 is proved.

\section{Acknowledgements}

The work of N.H. is partially supported by JSPS KAKENHI Grant Numbers JP20K03680, JP19H05597. The work of P.I.N. is partially supported by CONACYT project 283698 and PAPIIT project IN103221.

Data availability Data sharing is not applicable to this article as no new data were created or analyzed in this study.

Open Access. This article is licensed under a Creative Commons Attribution 4.0 International License, which permits use, sharing, adaptation, distribution and reproduction in any medium or format, as long as you give appropriate credit to the original author(s) and the source, provide a link to the Creative Commons licence, and indicate if changes were made. The images or other third party material in this article are included in the article's Creative Commons licence, unless indicated otherwise in a credit line to the material. If material is not included in the article's Creative Commons licence and your intended use is not permitted by statutory regulation or exceeds the permitted use, you will need to obtain permission directly from the copyright holder. To view a copy of this licence, visit http://creativecommons.org/licenses/by/4.0/.

Publisher's Note Springer Nature remains neutral with regard to jurisdictional claims in published maps and institutional affiliations.

\section{References}

[1] Bona, J.L., Saut, J.-C.: Dispersive blow-up of solutions of generalized Korteweg-de Vries equation. J. Differ. Equ. 103, 3-57 (1993)

[2] Bona, J.L., Ponce, G., Saut, J.-C., Sparber, C.: Dispersive blow-up for nonlinear Schrödinger equations revisited. J. Math. Pures Appl. (9) 102(4), 782-811 (2014)

[3] Calderon, A.P., Vaillancourt, R.: A class of bounded pseudo-differential operators. Proc. Natl. Acad. Sci. USA 69, $1185-1187(1972)$

[4] Carvajal, X.: Local well-posedness for a higher order nonlinear Schrödinger equation in Sobolev spaces of negative indices. Electron. J. Differ. Equ. 13, 1-13 (2004)

[5] Carvajal, X., Linares, F.: A higher order nonlinear Schrödinger equation with variable coefficients. Differ. Integral Equ. 16, 1111-1130 (2003)

[6] Cazenave, Th.: Semilinear Schrödinger equations, Courant Institute of Mathematical Sciences, New York; American Mathematical Society, Providence, RI, pp xiv+323 (2003)

[7] Coifman, R.R., Meyer, Y.: Au dela des operateurs pseudo-differentiels, p. 185. Societe Mathematique de France, Paris (1978)

[8] Constantin, P., Saut, J.-C.: Local smoothing properties of dispersive equations. J. Am. Math. Soc. 1, 413-446 (1988)

[9] Cordes, H.O.: On compactness of commutators of multiplications and convolutions, and boundedness of pseudodifferential operators. J. Funct. Anal. 18, 115-131 (1975)

[10] Craig, W., Kapeller, K., Strauss, W.A.: Gain of regularity for solutions of KdV type. Ann. Inst. Henri Poincare, Analyse non lineaire, 9, 147-186 (1992)

[11] de Bouard, A., Hayashi, N., Kato, K.: Gevrey regularizing effect for the (generalized) Korteweg-de Vries equation and nonlinear Schrödinger equations. Ann. Inst. Henri Poincare Analyse non lineaire 12, 673-725 (1995)

[12] Diaz-Otero, F.J., Chamorro-Posada, P.: Interchannel soliton collisions in periodic dispersion maps in the presence of third order dispersion. J. Nonlinear Math. Phys. 15, 137-143 (2008)

[13] Fedoryuk, M.V.: Asymptotic methods in analysis. In: Analysis. I. Integral representations and asymptotic methods. Encyclopaedia of Mathematical Sciences, 13. Springer, Berlin, (1989). vi+238 pp

[14] Hasegawa, A., Kodama, Y.: Nonlinear pulse propagation in a monomode dielectric guide. IEEE J. Quantum Electron. 23, 510-524 (1987) 
[15] Hayashi, N.: Analyticity of solutions of the Korteweg-de Vries equation. SIAM J. Math. Anal. 22, 1738-1745 (1991)

[16] Hayashi, N., Naumkin, P.I.: Asymptotics in large time of solutions to nonlinear Schrödinger and Hartree equations. Am. J. Math. 120, 369-389 (1998)

[17] Hayashi, N., Naumkin, P.I.: Domain and range of the modified wave operator for Schrödinger equations with a critical nonlinearity. Commun. Math. Phys 267, 477-492 (2006)

[18] Hayashi, N., Naumkin, P.I.: The initial value problem for the cubic nonlinear Klein-Gordon equation. Z. Angew. Math. Phys. 59(6), 1002-1028 (2008)

[19] Hayashi, N., Naumkin, P.: On the inhomogeneous fourth-order nonlinear Schrödinger equation. J. Math. Phys. 56(9), $093502(2015)$

[20] Hayashi, N., Kaikina, E.I.: Asymptotics for the third-order nonlinear Schrödinger equation in the critical case. Math. Methods Appl. Sci. 40(5), 1573-1597 (2017)

[21] Hayashi, N., Ozawa, N.: Scattering theory in the weighted $L^{2}\left(R^{n}\right)$ spaces for some Schrödinger equations. Ann. I.H.P. (Phys. Théor.) 48, 17-37 (1988)

[22] Karpman, V.I.: Stabilization of soliton instabilities by higher-order dispersion: fourth order nonlinear Schrödinger-type equations. Phys. Rev. E 53(2), 1336-1339 (1996)

[23] Kato, T.: On the Cauchy problem for the (generalized) Korteweg-de Vries equation. Advances in Mathematics Supplementary Studies Studies in Applied Mathematics, vol. 8, pp. 93-128, Berlin (1983)

[24] Kenig, C.E., Ponce, G., Vega, L.: On the (generalized) Korteweg-de Vries equation. Duke Math. J. 59, 585-610 (1989)

[25] Kenig, C.E., Ponce, G., Vega, L.: Well-posedness of the initial value problem for the Korteweg-de Vries equation. J. Am. Math. Soc. 4, 323-347 (1991)

[26] Kenig, C.E., Ponce, G., Vega, L.: Well-posedness and scattering results for the generalized Korteweg-de Vries equation via contraction principle. Commun. Pure Appl. Math. 46, 527-620 (1993)

[27] Kodama, Y.: Optical solitons in a monomode fiber. J. Stat. Phys. 39, 597-614 (1985)

[28] Kruzhkov, S.N., Faminskii, A.V.: Generalized solutions of the Cauchy problem for the Korteweg-de Vries equation. Math. USSR, Sbornik 48, 391-421 (1984)

[29] Laurey, C.: The Cauchy problem for a third order nonlinear Schrödinger equation. Nonlinear Anal. 29, 121-158 (1997)

[30] Mamyshev, P.V.: Generation and compression of femtosecond solitons in optical fibers. In: Taylor, J.R. (ed.) Optical Solitons-Theory and Experiment. Cambridge Studies in Modern Optics, Vol. 10, pp. 266-313. Cambridge University Press, Cambridge (1992)

[31] Mussot, A., Kudlinski, A., Louvergneaux, E., Kolobov, M., Taki, M.: Impact of the third-order dispersion on the modulation instability gain of pulsed signals. Opt. Lett. 35, 1194-1196 (2010)

[32] Ozawa, T.: Long range scattering for nonlinear Schrödinger equations in one space dimension. Commun. Math. Phys. 139(3), 479-493 (1991)

[33] Saut, J.-C.: Sur quelque generalisations de l'equation de Korteweg-de Vries. J. Math. Pure Appl. 58, 21-61 (1979)

[34] Taki, M., Mussot, A., Kudlinski, A., Louvergneaux, E., Kolobov, M., Douay, M.: Third-order dispersion for generating optical rogue solitons. Phys. Lett. A 374, 691-695 (2010)

Nakao Hayashi

Research Alliance Center for Mathematical Sciences

Tohoku University

Sendai 980-8578

Japan

e-mail: nakao.hayashi.e6@tohoku.ac.jp

Pavel I. Naumkin

Centro de Ciencias Matemáticas

UNAM Campus Morelia

AP 61-3 (Xangari)

CP 58089 Morelia Michoacán

Mexico

e-mail: pavelni@matmor.unam.mx

(Received: May 1, 2021; revised: September 14, 2021; accepted: October 8, 2021) 